\title{
TRAUMATIC IMPLANTATION CYST IN ANTERIOR CHAMBER AFTER CATARACT EXTRACTION*
}

\author{
BY
}

\author{
J. R. WHEELER \\ Belfast
}

\section{Case Report}

A male labourer, aged 74, attended the Royal Victoria Hospital, Belfast, complaining of defective vision.

Examination.- In the right eye, the visual acuity was reduced to 6/18, because of lens opacities. The left eye showed advanced lens opacities, and the visual acuity was good perception of light.

Operation.-On August 4, 1952, an intracapsular extraction of the opaque left lens was performed with a small total iridectomy, and a sclero-corneal silk suture at 12 o'clock closed the wound. Convalescence was uneventful. On September 16, 1952, 6 weeks after operation, the eye was quiescent, with media clear and fundus normal. Visual acuity with glasses was $6 / 6$ and $\mathrm{J} 1$.

Further Developments.-The patient was next seen 23 months later (July 6, 1954). Visual acuity in the left eye was now 1/60. A triangular deep striate keratitis was present with its base at the limbus from 1 o'clock to 3 o'clock and its apex involving the pupillary area. About half of the anterior chamber was filled by a large transparent cyst which seemed to arise from the limbal area around 2 o'clock (Fig. 1). The ocular tension was $23 \mathrm{~mm}$. Hg (Schiötz).

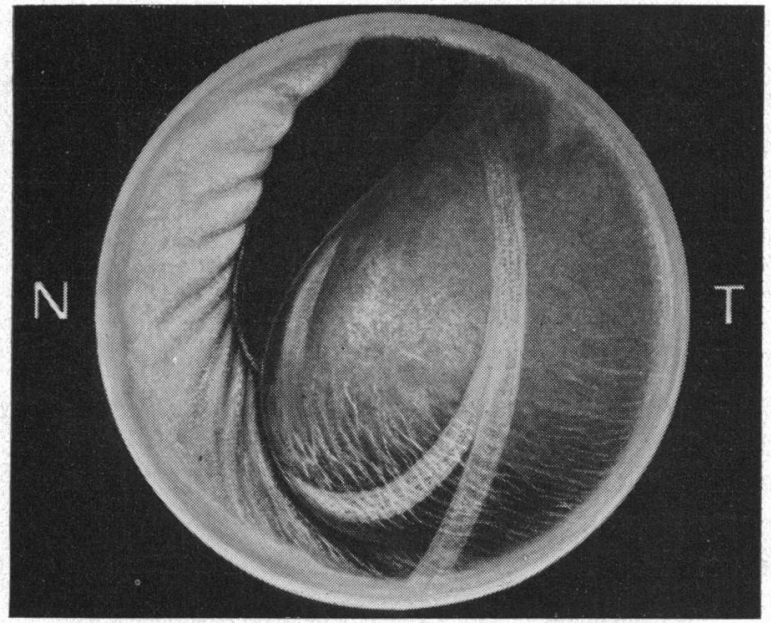

Fig. 1.-Left eye, July 6, 1954, slit-lamp view of cyst.

According to one authority the prognosis in such a case is "Gloomy-if allowed to progress eventually detroys eye through severe iridocyclitis and glaucoma". Another authority states that "Epitheliation of anterior chamber is a destructive lesion and calls for drastic treatment".

-Received for publication September 13, 1955. 
Treatment.-On July 15, 1954, I operated on the left eye. The anterior chamber was opened by a keratome incision at the limbus stretching from 10 o'clock to 1 o'clock. An iris repositor was passed in front of and behind the cyst, and it was established that its base was about $4 \mathrm{~mm}$. long and in line with the old "cataract" incision. An attempt was made to coax the cyst out of the anterior chamber by a vectis; when about half way out it burst and no evidence of it could be defined. The incision into the anterior chamber was now enlarged to include the area from which the cyst was arising. The base of the iris was removed from the involved area and the wound edges touched with a sharp pointed diathermy needle. The anterior chamber was washed out with penicillin and the wound edges were allowed to fall together. About 3 weeks later, in consultation with Dr. Millen, our radiotherapist, one radon seed was sewn to the conjunctiva at the limbus. A dosage of $4,000 \mathrm{r}$ at $0.5 \mathrm{~cm}$. was given. 6 months later the cyst had not recurred but keratitis caused the visual acuity to be much reduced.

\section{Discussion}

These cysts are formed by the active ingrowth of epithelium along the track of a perforating wound (surgical or traumatic). There are two main types:

(a) Pearl cysts which are solid looking

(b) Translucent cysts with thin walls.

The second type-the translucent cysts-are more common and are composed of relatively thin-walled, ill-developed epithelium containing a yellow fluid.

Apparently the first cases on record were reported by Collins and Cross (1892). Two theories were put forward regarding the mode of formation of these cysts, the authors inclining towards the latter:

(1) The epithelium arose from proliferation of the 'endothelium lining Descemet's membrane and the anterior surface of the iris;

(2) Surface epithelium was carried in at the time of operation and subsequently proliferated.

Carrado (1931) found by experiments on animals that only when the corneal wound remains open beyond a certain time is it possible for corneal epithelium, by proliferation, to cover the border of the wound and to penetrate into the anterior chamber. This is facilitated by incarceration of the iris, lens capsule, or vitreous in the wound. He found it was not sufficient for a strip of epithelial tissue to fall into the anterior chamber for attachment and proliferation to occur.

The histology of the diseased process has been described by Parsons (1904) as follows:

On reaching the back of the cornea the epithelium insinuates itself between Descemet's membrane and substantia propria and also grows on the posterior surface. A variable number of layers of cells are found, the lowest cells being cylindrical or cubical, the highest very flat and long. The same applies when the iris is covered or the whole chamber lined; the epithelium varies extremely in thickness, from a single layer to five or six or more and the cells are found ill formed. 
From the clinical point of view there would appear to be three potential precursors to this cystic formation:

(1) Delayed formation of anterior chamber;

(2) A leaking wound;

(3) Fistulization of the wound.

There is no doubt that the accurate closure of the corneo-scleral incision for cataract by well-placed sutures will help to obtain an early and firmly healed wound and so prevent fistulization. This has been made possible during the last decade by the introduction of beautifully fine cutting needles threaded with $6 / 0$ silk. There are various methods of suturing but personally .I like to place my sclero-corneal suture in position and then deliberately cut through it with my cataract knife (Fig. 2). The two cut ends of the

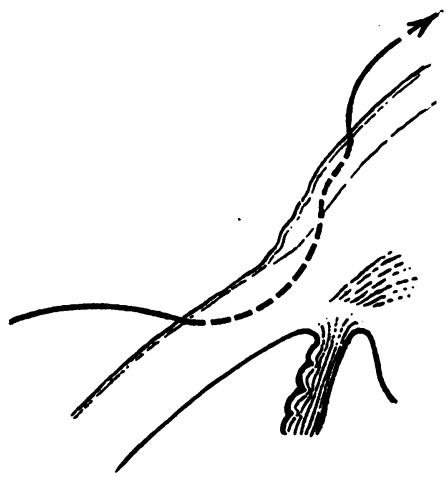

(a). Suture in position before incision is made.

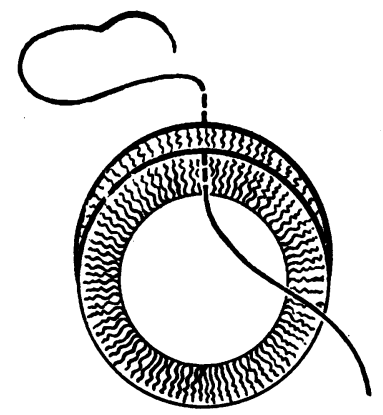

(b). Position of suture after incision.

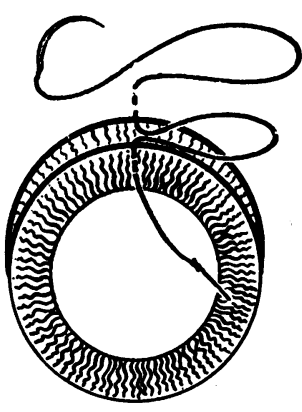

(c). Original suture withdrawn and new suture inserted before extraction commences.

Fig. 2.-Method of inserting sutures.

suture are easily seen and a new suture can be readily passed through the track as one's assistant withdraws the cut ends. By this means exact apposition of the wound edges is achieved. In order that the wound may be closed immediately after delivery of the lens I tie a knot on the corneal arm of the suture. My assistant can now pull on the scleral arm of the suture, against the knot, and so close the wound immediately behind the " departing" lens. The stitch should be tied firmly, not tightly. Lateral sutures may be inserted if the wound appears to bulge or if the patient is of a hyperkinetic type.

\section{REFERENCES}

Corrado, M. (1931). Ann. Ottal., 59, 706.

Cross, F. Richardson, and Collins, E. Treacher (1892). Brit. med. J., 2, 130.

Parsons, J. H. (1904). " The Pathology of the Eye ", vol. 1, p. 151. Hodder and Stoughton, London. 